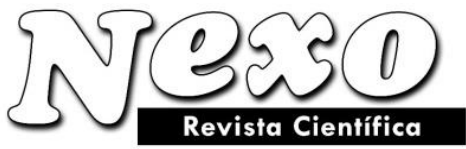

ISSN-E 1995-9516

Universidad Nacional de Ingeniería COPYRIGHT () (UNI). TODOS LOS DERECHOS RESERVADOS http://revistas.uni.edu.ni/index.php/Nexo https://doi.org/10.5377/nexo.v34i06.13115

\title{
Diseño térmico de un economizador de tubos aleteados
}

\section{Thermal design of a finned tubes economizer}

\author{
Amaury Pérez Sánchez ${ }^{1} *$, Noemí Acosta Pérez², Greisy I. Valero Almanza ${ }^{3}$, Elizabeth Ranero \\ González ${ }^{1}$, Eddy J. Pérez Sánchez ${ }^{4}$ \\ ${ }^{1}$ Universidad de Camagüey "Ignacio Agramonte Loynaz", Facultad de Ciencias Aplicadas, Departamento \\ de Ingeniería Química, Camagüey, Cuba. \\ ${ }^{2}$ Empresa Azucarera de Camagüey, Laboratorio Físico-Químico, Camagüey, Cuba. \\ ${ }^{3}$ Centro de Ingeniería Ambiental de Camagüey, Departamento de Gestión Ambiental, Camagüey, Cuba. \\ ${ }^{4}$ Empresa Servicios Automotores S.A., Dirección Comercial, Ciego de Ávila, Cuba. \\ *amaury.perez84@gmail.com
}

(recibido/received: 25-junio-2021; aceptado/accepted: 07-agosto-2021)

\section{RESUMEN}

Un economizador es un intercambiador de calor de tubos aleteados que permite recuperar parte del calor sensible de los productos de la combustión que emite una caldera, transfiriéndolo al agua de alimentación y aumentando así la eficiencia térmica. En el presente trabajo se efectuó el diseño de un economizador de tubos con aletas helicoidales para efectuar el precalentamiento de un agua de alimentación que se encuentra a una temperatura de $25^{\circ} \mathrm{C}$, utilizando para ello gases de combustión a $240{ }^{\circ} \mathrm{C}$ como agente de transferencia de calor, los cuales se emiten al quemar Fuel-Oil en una caldera pirotubular. El agua se calentará hasta $73,27{ }^{\circ} \mathrm{C}$, y tanto el coeficiente global de transferencia de calor como el área de la superficie de transferencia de calor tuvieron valores de $19,947 \mathrm{~W} / \mathrm{m}^{2} . \mathrm{K}$ y $95,146 \mathrm{~m}^{2}$, respectivamente. El economizador diseñado tendrá una longitud de los tubos aleteados de 65,482 m, un número total de tubos de 131, un número de filas transversales de los tubos de 16, una profundidad del conducto del gas de $0,765 \mathrm{~m}$, un número real de tubos de 144 y una longitud actual de los tubos aleteados igual a 72 .

Palabras claves: Agua; Diseño térmico; Economizador; Gases de combustión; Tubos aleteados.

\section{ABSTRACT}

An economizer is a finned tube heat exchanger that makes it possible to recover part of the sensible heat of the combustion products emitted by a boiler, transferring it to the feed water and thus increasing thermal efficiency. In the present work, the design of an economizer of tubes with helical fins was carried out to accomplish the preheating of a feed water that is at a temperature of $25^{\circ} \mathrm{C}$, by using combustion gases at $240{ }^{\circ} \mathrm{C}$ as heat transfer agent which are emitted by burning Fuel-Oil in a fire tube boiler. The water will heat up to $73.27^{\circ} \mathrm{C}$, and both the global heat transfer coefficient and the heat transfer surface area had values of $19.947 \mathrm{~W} / \mathrm{m}^{2} . \mathrm{K}$ and $95.146 \mathrm{~m}^{2}$, respectively. The economizer designed will have a length of the finned tubes of $65.482 \mathrm{~m}$, a total number of tubes of 131, a number of transverse rows of tubes of 16, a depth of the gas conduit of $0.765 \mathrm{~m}$, an actual number of tubes of 144 and an actual length of the finned tubes equal to 72 . 
Keywords: Water; Thermal design; Economizer; Combustion gases; Finned tubes.

\section{NOMENCLATURA}

a

A

A'

$\mathrm{A}_{1}$

$\mathrm{A}_{\text {in } 1}$

$\mathrm{A}_{\text {in }}$

$\mathrm{A}_{\text {in }}^{\prime}$

$\mathrm{A}_{\mathrm{r} 1}$

$\mathrm{A}_{\text {rct1 }}$

At1

b

c

$\mathrm{Cp}$

$\mathrm{C}_{\mathrm{q}}$

$\mathrm{C}_{\text {tem }}$

$\mathrm{C}_{\mathrm{z}}$

d

$\mathrm{d}_{\mathrm{cl}}$

$\mathrm{d}_{\text {in }}$

D

E

E'

f

F

G

h'

h'

$\mathrm{h}_{1 \mathrm{rdc}}$

h'

$\mathrm{h}_{2}$

$\mathrm{h}_{\mathrm{c}}$

$\mathrm{k}$

$l_{\mathrm{r}}$

$l_{r}^{\prime}$

$L_{r s}^{a}$

$\mathrm{L}_{\mathrm{c} \cdot \mathrm{cr} \cdot \mathrm{s}}$

$\mathrm{L}_{\mathrm{rs}}$

$\mathrm{L}_{\mathrm{t}}$

$\mathrm{n}$

$\mathrm{n}_{\mathrm{x}}$

$\mathrm{P}^{\prime}$

$\operatorname{Pr}$

Q

$\mathrm{R}_{\text {cont }}$
Ancho del conducto de gas

Área de la superficie de transferencia de calor del intercambiador de calor

Área asumida de la superficie exterior de transferencia de calor

Área de la superficie externa por $1 \mathrm{~m}$ de longitud del tubo

Área de la superficie interna por $1 \mathrm{~m}$ de longitud del tubo

Área de la superficie de transferencia de calor interna calculada

Área interior calculada

Área superficial de las aletas por $1 \mathrm{~m}$ de longitud del tubo para aletas helicoidales

Área superficial total del tubo portador aleteado por $1 \mathrm{~m}$ de longitud del tubo

Área superficial del tubo portador, no ocupada por las aletas, por $1 \mathrm{~m}$ de longitud del

tubo aleteado

Altura del conducto de gas

Profundidad del conducto de gas

Calor especifico

Factor

Factor de corrección

Factor

Diámetro exterior del tubo portador de la aleta

Diámetro convencional del tubo aleteado

Diámetro interior del tubo

Diámetro del tubo aleteado

Eficiencia teórica de la aleta

Valor preliminar de la eficiencia teórica de la aleta

Área de flujo para el paso del medio de transferencia de calor

Área libre mínima

Caudal másico

Entalpia de entrada del fluido frío

Entalpia de salida del fluido frío

Coeficiente de transferencia de calor reducido desde el exterior de la superficie de calentamiento

Coeficiente de transferencia de calor desde la pared hacia el medio interno asumido

Coeficiente de transferencia de calor desde la pared hacia el medio interno

Coeficiente de transferencia de calor convectivo

Conductividad térmica

Altura de la aleta

Altura convencional de la aleta

Longitud actual de los tubos aleteados del intercambiador de calor

Longitud de los tubos dentro de los confines del conducto de gas

Longitud de los segmentos aleteados de los tubos

Longitud total de los segmentos calentados de los tubos no aleteados

Exponente

Numero de comienzos del serpentín

Presión de entrada

Número de Prandtl

Calor intercambiado

Resistencia térmica de contacto en la interface acero-aluminio $\mathrm{m}$

$\mathrm{m}^{2}$

$\mathrm{m}^{2}$

$\mathrm{m}^{2}$

$\mathrm{m}^{2}$

$\mathrm{m}^{2}$

$\mathrm{m}^{2}$

$\mathrm{m}^{2}$

$\mathrm{m}^{2}$

$\mathrm{m}^{2}$

m

$\mathrm{m}$

$\mathrm{kJ} / \mathrm{kg} . \mathrm{K}$

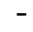

-

$\mathrm{m}$

$\mathrm{m}$

$\mathrm{m}$

$\mathrm{m}$

-

$\mathrm{m}^{2}$

$\mathrm{m}^{2}$

$\mathrm{kg} / \mathrm{s}$

$\mathrm{kJ} / \mathrm{kg}$

$\mathrm{kJ} / \mathrm{kg}$

$\mathrm{W} / \mathrm{m}^{2} . \mathrm{K}$

$\mathrm{W} / \mathrm{m}^{2} . \mathrm{K}$

$\mathrm{W} / \mathrm{m}^{2} . \mathrm{K}$

$\mathrm{W} / \mathrm{m}^{2} . \mathrm{K}$

$\mathrm{W} / \mathrm{m} . \mathrm{K}$

$\mathrm{m}$

$\mathrm{m}$

$\mathrm{m}$

$\mathrm{m}$

$\mathrm{m}$

$\mathrm{m}$

-

$\mathrm{Pa}$

$\mathrm{kW}$

$\mathrm{m}^{2} . \mathrm{K} / \mathrm{W}$ 
$\mathrm{Re}$
$\mathrm{R}_{\mathrm{T}}$
$\mathrm{S}_{\mathrm{r}}$
$\mathrm{S}_{1}$
$\mathrm{~S}_{2}$
$\mathrm{~S}_{2}{ }_{2}$
$\mathrm{~T}^{\prime}$
$\mathrm{T}^{\prime}$
$\bar{T}$
$\mathrm{~T}_{\text {in }}$
$\mathrm{T}_{\mathrm{r}}$
$\Delta \mathrm{T}$
$\mathrm{u}$
$\mathrm{U}$
$\mathrm{v}$
$\mathrm{X}$
$\mathrm{Z}$
$\mathrm{Z}_{1}$
$\mathrm{Z}_{2}$
$z^{a}$
$\mathrm{Z}_{\text {tcp }}$

$\beta$

$\delta_{\mathrm{r}}$

$\delta^{\prime}{ }_{t}$

$\varepsilon$

$\zeta$

$\lambda$

$\mu$

$\mu_{\mathrm{r}}$

$\rho$

$\sigma_{1}$

$\sigma_{2}$

$\varphi_{\mathrm{cl}}$

$\Psi$

$\Psi_{\mathrm{E}}$

$\Psi_{\mathrm{r}}$

$\vartheta$

9,

$\bar{\vartheta}$

f

g

$\mathrm{r}$
Número de Reynolds

Resistencia térmica de la pared de los tubos bimetálicos

Espaciado de las aletas

Espaciado transversal del tubo

Espaciado longitudinal del tubo

Espaciado diagonal del tubo con un arreglo "equivalente"

Temperatura de entrada del fluido frío

Temperatura de salida del fluido frío

Temperatura promedio del fluido frío

Temperatura interior

Temperatura promedio de la aleta

Diferencia promedio de temperatura

Velocidad

Coeficiente global de transferencia de calor

Viscosidad cinemática

Parámetro de forma del haz

Número de tubos en el haz

Número de tubos en la fila transversal del haz

Numero de filas de tubos en el haz en dirección al flujo de gas

Número real de tubos en el intercambiador de calor

Número de tubos conectados en paralelo

\section{Símbolos griegos}

Parámetro de la aleta

Espesor medio de las aletas

Espesor nominal de la pared del tubo

Volumen específico

Parámetro

Parámetro

Viscosidad dinámica

Coeficiente

Densidad

Espaciado transversal relativo de los tubos en el haz

Espaciado longitudinal relativo de los tubos en el haz

Diámetro del haz

Eficiencia térmica

Factor

Coeficiente de la aleta del tubo

Temperatura de entrada del fluido caliente

Temperatura de salida del fluido caliente

Temperatura promedio del fluido caliente

\section{Subíndices}

Del líquido

Del gas

De la aleta $\mathrm{m}^{2} \cdot \mathrm{K} / \mathrm{W}$

$\mathrm{m}$

$\mathrm{m}$

$\mathrm{m}$

$\mathrm{m}$

${ }^{\circ} \mathrm{C}$

${ }^{\circ} \mathrm{C}$

${ }^{\circ} \mathrm{C}$

${ }^{\circ} \mathrm{C}$

${ }^{\circ} \mathrm{C}$

${ }^{\circ} \mathrm{C}$

$\mathrm{m} / \mathrm{s}$

$\mathrm{W} / \mathrm{m}^{2} . \mathrm{K}$

$\mathrm{m}^{2} / \mathrm{s}$

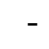

-

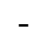

-

-

-

m

$\mathrm{m}$

$\mathrm{m}^{3} / \mathrm{kg}$

-

Pa.s

Pa.s

$\mathrm{kg} / \mathrm{m}^{3}$
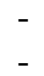

-

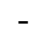

-

$-$

${ }^{\circ} \mathrm{C}$

${ }^{\circ} \mathrm{C}$

${ }^{\circ} \mathrm{C}$

\section{INTRODUCCIÓN}

El incremento continuo de la demanda de energía asociado al desarrollo socioeconómico, las restricciones financieras para ampliar la oferta energética, la necesidad de lograr una mayor competitividad 
internacional y de atenuar el impacto ambiental de las tecnologías energéticas, fundamentan la importancia que tiene hoy en día el mejoramiento de la eficiencia energética de cualquier proceso (Restrepo et al., 2006).

La creciente competencia que existe hoy día obliga a las empresas a buscar alternativas para reducir los costos operacionales de sus procesos productivos (Oelker, 1999). Los consumos elevados de combustible y sus altos costos, así como, la problemática de la contaminación atmosférica, han llevado a buscar métodos que permitan el uso racional y eficiente de la energía.

Una caldera o generador de vapor es un equipo destinado a la producción de vapor a partir de agua líquida. La energía necesaria para producir el vapor de agua se obtiene de las reacciones de combustión de un combustible sólido, líquido o gas, conjuntamente con el oxígeno del aire. Los gases calientes provenientes de las reacciones químicas se expulsan al medio ambiente. Es posible utilizar los gases para precalentar el agua fría que ingresa a la caldera mediante el empleo de un intercambiador de calor denominado economizador (Armijo y Salas, 2008).

La temperatura de los gases de combustión que salen por la chimenea representa uno de los mayores porcentajes de pérdidas generados en la caldera. Éstas pérdidas de calor asociada a los productos (gases) de la combustión (en chimenea) representa un alrededor de un $18 \%$ para el caso de las calderas que utilizan gas y $12 \%$ para el caso de las calderas que utilizan carbón y petróleo. En la mayoría de los casos, dado que la temperatura de salida de los gases de la combustión es de $230{ }^{\circ} \mathrm{C}$ como mínimo, es posible recuperar el calor sensible de estos gases, incluso hasta $170{ }^{\circ} \mathrm{C}$ (caso Fuel-Oil), instalando un economizador al final del recorrido de los gases por la caldera, que calienta el agua de alimentación de la misma (Oelker, 2000).

La temperatura del agua de alimentación es muy importante en la caldera. Teóricamente está relacionado con la cantidad de calor requerido para el calentamiento del agua (sensible) y el cambio de fase (latente). Según (Chucuya, 2017) la eficiencia de la caldera aumenta con la elevación de la temperatura del agua de alimentación al necesitarse menos energía para producir el vapor. Además, añade que como "regla de dedo", al aumentar $5^{\circ} \mathrm{C}$ la temperatura de alimentación de agua a la caldera implicará un aumento del $1 \%$ en la eficiencia de la caldera.

Los economizadores son básicamente superficies de transferencia de calor tubulares usadas para precalentar el agua de alimentación a las calderas antes que entren al tambor de vapor (unidades de recirculación) o superficies de horno (unidades de un solo paso). El término economizador proviene del uso temprano de tales intercambiadores de calor para reducir los costos de operación o economizar el consumo de combustible mediante la recuperación de energía extra a partir de los gases de combustión. Los economizadores también reducen el potencial de choque térmico y las fuertes fluctuaciones de la temperatura del agua a medida que el agua de alimentación entra hacia el tambor o las paredes de agua (Brumerčík et al., 2016).

De acuerdo con (Farthing, 2008), los economizadores son aparatos térmico-mecánicos que depuran el calor residual contenido en los gases de combustión haciendo pasar el efluente de escape a través de superficies de transferencia de calor para transferir algo del calor residual hacia un fluido de proceso. En el hogar de la caldera la mayoría de los economizadores transfieren su calor residual hacia tanto el agua de alimentación como el aire de combustión. Un economizador de agua de alimentación (Fig. 1) es una de las adiciones más económicas que pueden hacerse para cualquier caldera. La tecnología sencilla de los economizadores y la falta de partes en movimiento le suministra un ciclo de vida relativamente elevado libre de mantenimiento. Resulta necesario apuntar que los economizadores se emplean con estrategias de alimentación de agua modulantes para prevenir el sobrecalentamiento y el recalentamiento repentino, el cual puede ocurrir en ciertas aplicaciones. 


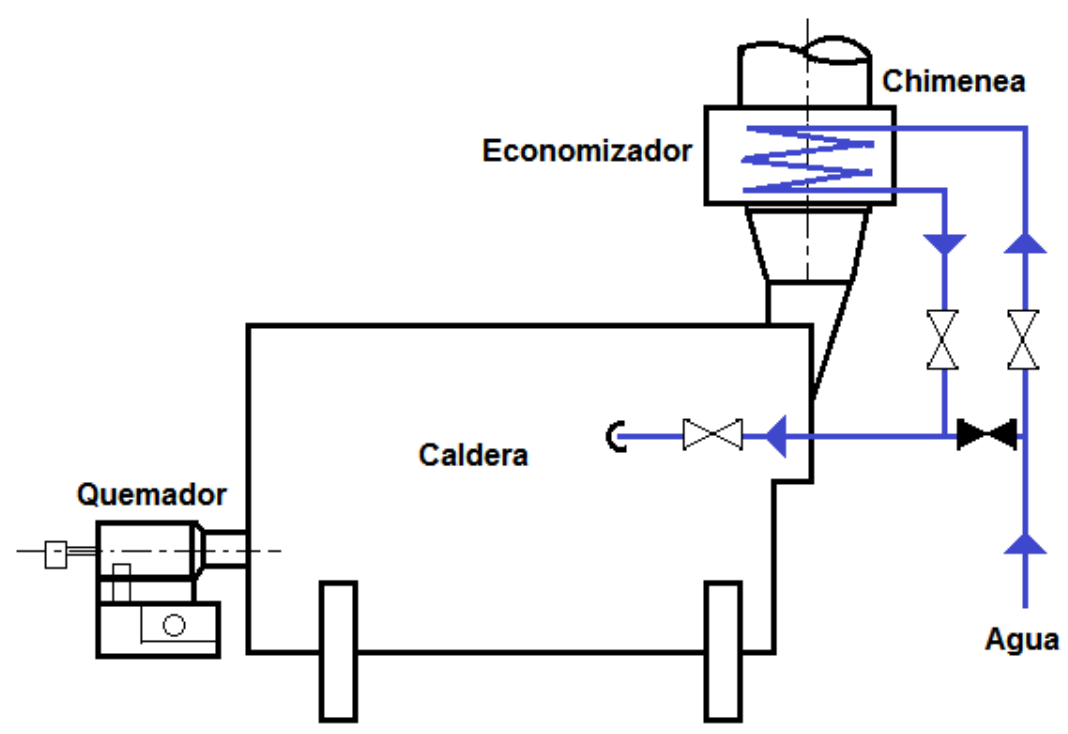

Figure 1. Esquema de instalación de un economizador en una caldera.

Fuente: Adaptado de (Oelker, 1999)

Para reducir los costos de capital, la mayoría de los fabricantes de calderas construyen economizadores con una variedad de tipos de aletas para mejorar la tasa de transferencia de calor controlante del lado del gas. Las aletas son partes no costosas las cuales pueden reducir el tamaño global y el costo de un economizador. Sin embargo, la aplicación exitosa es muy sensible al ambiente reinante cuando se emplean gases de combustión. La limpieza de la superficie es una preocupación clave. En calderas escogidas, tales como aquellas unidades que queman carbón, los economizadores de superficie extendida no son recomendables debido a las características peculiares del polvo de ceniza (Brumerčík et al., 2016).

La principal ventaja o beneficio de un economizador es la importante reducción de los gastos en combustible, resultante del mejoramiento de la eficiencia de las calderas, lo que permite quemar una menor cantidad de combustible para aportar el calor requerido para generar vapor. El hecho de requerir de una menor cantidad de combustible se traduce también en una reducción de las emisiones contaminantes de una caldera, puesto que éstas dependen de la cantidad de combustible quemado. También evitará los choques térmicos producidos cuando el agua de alimentación a baja temperatura entra en contacto con las superficies calientes de la caldera (Oelker, 1999).

Son varios los autores que han estudiado, evaluado o diseñado economizadores. En este sentido (Martić et al., 2015), presentaron un análisis técnico y económico de una planta pequeña de cogeneración que se construyó para suministrar tanto agua caliente como vapor de agua para una planta anexa de procesamiento lácteo en Serbia, presentando además una metodología para diseñar un economizador. También (Brumerčík et al., 2016) emplearon la modelación de Método de Elemento Finito para determinar los campos de tensión y temperaturas de los componentes de un economizador. Igualmente (Jatola et al., 2017) efectuaron el análisis de rendimiento de un economizador utilizando diferentes materiales de los tubos. Por otro lado, (Trojan y Granda, 2018) presentaron un modelo matemático de un economizador con parámetros distribuidos, el cual puede usarse para simular su operación. El modelo matemático desarrollado por estos autores hace posible determinar las temperaturas del tubo y del medio de trabajo, así como también permite monitorear los parámetros de calor y flujo del economizador en modo online. De igual manera, (Chopade, 2018) efectuó el diseño de un economizador para ser empleado en una caldera recuperadora de calor residual para mejorar la capacidad térmica del proceso. Asimismo, (Khoirul y Firman, 2019) diseñaron un intercambiador de calor de tubo y coraza para ser empleado como economizador, determinando parámetros tales como el área de sección transversal, la longitud del tubo, el 
diámetro exterior del tubo y el número total de tubos. Estos autores optimizaron las principales dimensiones del intercambiador de calor por medio del método experimental de Taguchi para obtener el diseño óptimo. En otro estudio, (López, 2017) diseñó un economizador utilizando la metodología publicada en (Pysmenny et al., 2007). Por último, (Zarifi y Moghaddam, 2020) estudiaron el empleo de un economizador de tubos aleteados para incrementar la eficiencia de un generador termoeléctrico basado en un sistema de cogeneración.

Otros autores (Melendez, 2005; Morales y Veintimilla, 2007; Ruiz, 2012; Guiot y Ramos, 2018; Pacheco y Vargas, 2021) también han diseñado economizadores para diferentes aplicaciones y servicios.

En el presente trabajo se efectúa el diseño de un economizador de tubos aleteados para efectuar el calentamiento del agua de alimentación a una caldera, mediante el aprovechamiento de los gases de combustión generados en ésta, utilizando para ello la metodología presentada en (Pis'mennyi et al., 2016).

\section{MATERIALES Y MÉTODOS}

\subsection{Definición del problema}

Se necesita diseñar un economizador para efectuar el pre-calentamiento de $1,20 \mathrm{~kg} / \mathrm{s}$ de un agua de alimentación a una caldera. La temperatura y presión de entrada del agua es de $25{ }^{\circ} \mathrm{C}$ y $0,5 \mathrm{MPa}$, respectivamente, mientras que se emplearán los gases de combustión como agente de intercambio térmico, los cuales se encuentran a una temperatura de $240{ }^{\circ} \mathrm{C}$ y presión de $1 \mathrm{~atm}$, y presentan la siguiente composición porcentual volumétrica:

- Dióxido de carbono (yco2): 10,17\%.

- Oxígeno ( $\left.\mathrm{y}_{\mathrm{O} 2}\right): 7,20 \%$.

- Nitrógeno ( $\left.\mathrm{y}_{\mathrm{N} 2}\right): 73,22 \%$.

- Agua (унго): 9,70\%.

- Dióxido de azufre (ysoz): 0,004\%.

- Monóxido de carbono (yсо): 0,006\%.

Se desea que la temperatura de salida de los gases de combustión no sobrepase los $120{ }^{\circ} \mathrm{C}$, que el economizador opere a contracorriente con arreglo triangular de los tubos, y que posea tubos con aletas helicoidales. Para ello se disponen de tubos aleteados bimetálicos con las siguientes características geométricas y dimensiones:

Tubo:

- Material: Acero al carbono.

- Diámetro interno $\left(\mathrm{d}_{\text {in }}\right): 0,02786 \mathrm{~m}$.

- Espesor de la pared $\left(\delta^{\prime}{ }_{\mathrm{t}}\right): 0,00277 \mathrm{~m}$.

- Diámetro externo (d): 0,0334 m.

Aletas:

- Material: Aluminio.

- Diámetro del tubo aleteado (D): 0,0604 m.

- Altura de la aleta $\left(1_{\mathrm{r}}\right): 0,0135 \mathrm{~m}$.

- Espaciado de las aletas $\left(\mathrm{s}_{\mathrm{r}}\right): 0,003 \mathrm{~m}$.

- Espesor medio de las aletas $\left(\delta_{\mathrm{r}}\right): 0,0008 \mathrm{~m}$.

Otros datos:

- Ancho del conducto de gas (a) (no mayor de): 0,56 m 
- Altura del conducto de gas (b) (no mayor de): 0,50 m.

- Longitud de los segmentos aleteados de los tubos $\left(\mathrm{L}_{\mathrm{rs}}\right): 1 \mathrm{~m}$.

- Número de tubos en el haz (z): 1.

- Longitud total de los segmentos calentados de los tubos no aleteados $\left(\mathrm{L}_{\mathrm{t}}\right): 0 \mathrm{~m}$.

- Número de tubos en la fila transversal del haz $\left(\mathrm{z}_{1}\right): 9$

- Numero de filas de tubos en el haz en dirección al flujo de gas $\left(\mathrm{z}_{2}\right): 10$

- Longitud de los tubos dentro de los confines del conducto de gas ( $\left.\mathrm{L}_{\mathrm{c} \cdot \mathrm{cr} \cdot \mathrm{s}}\right)$ : 0,5 m.

En la Figura 2 se muestra la nomenclatura empleada tanto en los datos iniciales como en los cálculos posteriores, para efectuar el diseño del economizador.
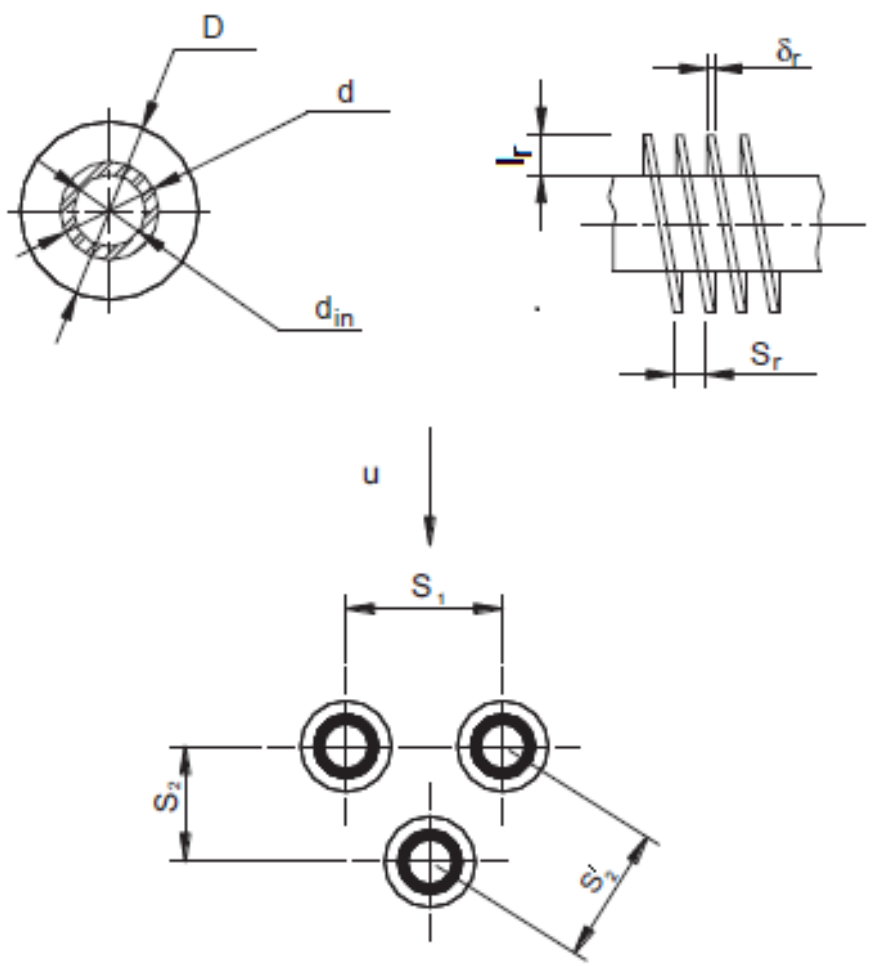

Figura 2. Características geométricas de los tubos aleteados y el haz de tubos con arreglo triangular. Fuente: Adaptado de (Pis'mennyi et al., 2016)

\subsection{Metodología de cálculo a emplear}

Para llevar a cabo el diseño del economizador se empleó la metodología publicada en (Pis'mennyi et al., 2016), en donde se obtiene como parámetros fundamentales el coeficiente global de transferencia de calor, el área total de la superficie de transferencia de calor, el número total de tubos, el número de filas transversales y el número real de tubos en el intercambiador de calor, así como también la longitud real de tubos aleteados y la profundidad del conducto de gas. A continuación, se muestran los pasos a seguir para efectuar el diseño del equipo.

Coeficiente de transferencia de calor convectivo:

Paso 1. Definición de los datos iniciales de las corrientes: 
La Tabla 1 muestra los datos iniciales que deben definirse para ambas corrientes de fluido.

Tabla 1. Datos iniciales a definir para ambas corrientes de fluido:

\begin{tabular}{llll}
\hline Parámetro & Agua & Gases & Unidades \\
\hline Caudal másico & $\mathrm{G}_{\mathrm{f}}$ & $\mathrm{G}_{\mathrm{g}}$ & $\mathrm{kg} / \mathrm{s}$ \\
Temperatura de entrada & $\mathrm{T}{ }^{\prime}$ & $\vartheta^{\prime}$ & ${ }^{\circ} \mathrm{C}$ \\
Temperatura de salida & - & $\vartheta^{\prime}$, & ${ }^{\circ} \mathrm{C}$ \\
Presión de entrada & $\mathrm{P}_{\mathrm{f}}$ & $\mathrm{P}_{\mathrm{g}}$ & $\mathrm{MPa}$ \\
\hline Fuente: Elaboración propia. & & &
\end{tabular}

Paso 2. Definición de los datos iniciales requeridos para el intercambiador de calor:

La Tabla 2 expone los datos iniciales requeridos para el intercambiador de calor

Tabla 2. Datos iniciales requeridos para el intercambiador de calor:

\begin{tabular}{|c|c|c|}
\hline Parámetro & Símbolo & Unidades \\
\hline \multicolumn{3}{|l|}{ Tubos } \\
\hline Diámetro interno & $\mathrm{d}_{\text {in }}$ & $\mathrm{m}$ \\
\hline Espesor de la pared & $\delta_{t}^{\prime}$ & $\mathrm{m}$ \\
\hline Diámetro externo & $\mathrm{d}$ & $\mathrm{m}$ \\
\hline \multicolumn{3}{|l|}{ Aletas } \\
\hline Diámetro del tubo aleteado & $\mathrm{D}$ & $\mathrm{m}$ \\
\hline Altura de la aleta & $1_{\mathrm{r}}$ & $\mathrm{m}$ \\
\hline Espaciado de las aletas & $\mathrm{S}_{\mathrm{r}}$ & $\mathrm{m}$ \\
\hline Espesor medio de las aletas & $\delta_{\mathrm{r}}$ & $\mathrm{m}$ \\
\hline \multicolumn{3}{|l|}{ Otros datos } \\
\hline Ancho del conducto de gas & $\mathrm{a}$ & $\mathrm{m}$ \\
\hline Altura del conducto de gas & $\mathrm{b}$ & $\mathrm{m}$ \\
\hline Longitud de los segmentos aleteados de los tubos & $\mathrm{L}_{\mathrm{rs}}$ & $\mathrm{m}$ \\
\hline Número de tubos en el haz & $\mathrm{Z}$ & \\
\hline Longitud total de los segmentos calentados de los tubos no aleteados & $\mathrm{L}_{\mathrm{t}}$ & $\mathrm{m}$ \\
\hline Número de tubos en la fila transversal del haz & $\mathrm{z}_{1}$ & \\
\hline Numero de filas de tubos en el haz en dirección al flujo de gas & $\mathrm{z}_{2}$ & \\
\hline Longitud de los tubos dentro de los confines del conducto de gas & $\mathrm{L}_{\mathrm{c} \cdot \mathrm{cr} \cdot \mathrm{s}}$ & $\mathrm{m}$ \\
\hline Resistencia térmica de contacto en la interface acero-aluminio & $\mathrm{R}_{\text {cont }}$ & $\mathrm{m}^{2} . \mathrm{K} / \mathrm{W}$ \\
\hline
\end{tabular}

Paso 3. Temperatura promedio de los gases de combustión $(\bar{\vartheta})$ :

$$
\bar{\vartheta}=\frac{\vartheta^{\prime}+\vartheta^{\prime \prime}}{2}
$$

Paso 4. Búsqueda de las propiedades físicas de los gases de combustión a la temperatura promedio determinada en el paso anterior:

Se deberán buscar las propiedades físicas descritas en la Tabla 3 para los gases de combustión a la temperatura promedio calculada en el Paso 3.

Tabla 3. Propiedades físicas que deben determinarse para los gases de combustión:

\begin{tabular}{lll}
\hline Propiedad & Símbolo Unidades \\
\hline
\end{tabular}




\begin{tabular}{lcc}
\hline Densidad & $\rho_{\mathrm{g}}$ & $\mathrm{kg} / \mathrm{m}^{3}$ \\
Volumen específico & $\varepsilon_{\mathrm{g}}$ & $\mathrm{m}^{3} / \mathrm{kg}$ \\
Viscosidad cinemática & $\mathrm{Vg}_{\mathrm{g}}$ & $\mathrm{m} / \mathrm{s}$ \\
Calor especifico & $\mathrm{Cp}_{\mathrm{g}}$ & $\mathrm{kJ} / \mathrm{kg} \cdot \mathrm{K}$ \\
Conductividad térmica & $\mathrm{kg}_{\mathrm{g}}$ & $\mathrm{W} / \mathrm{m} \cdot \mathrm{K}$ \\
Número de Prandtl & $\mathrm{Pr}_{\mathrm{g}}$ & - \\
\hline Fuente: Elaboración propia & &
\end{tabular}

Paso 5. Calor intercambiado (Q):

$$
Q=G_{g} \cdot C p_{g} \cdot\left(\vartheta^{\prime}-\vartheta^{\prime \prime}\right)
$$

Paso 6. Entalpía del agua a la entrada del intercambiador de calor (h'):

En base a las temperaturas $\left(\mathrm{T}^{\prime}\right)$ y presión $\left(\mathrm{P}_{\mathrm{f}}\right)$ de entrada del agua, deberá buscarse la entalpía del agua a la entrada del intercambiador de calor (h').

Paso 7. Entalpía del agua a la salida del intercambiador de calor (h’'):

A partir de la ecuación de balance:

$$
Q=G_{f} \cdot\left(h^{\prime \prime}-h^{\prime}\right)
$$

Despejando h"' en la ecuación (3) se obtiene:

$$
h^{\prime \prime}=h^{\prime}+\frac{Q}{G_{f}}
$$

Paso 8. Temperatura del agua a la salida del intercambiador de calor ( $T$ ' '):

Tomando en cuenta el valor de tanto la presión de alimentación del agua ( $\mathrm{P}_{\mathrm{f}}$ ) como su entalpía de salida (h"), se puede definir la temperatura del agua a la salida del intercambiador de calor, esto es, hasta que temperatura se calentará esta agua.

Paso 9. Temperatura promedio del agua en el intercambiador de calor $(\bar{T})$ :

$$
\bar{T}=\frac{T^{\prime}+T^{\prime \prime}}{2}
$$

Paso 10. Búsqueda de las propiedades físicas del agua de alimentación:

Tomando en cuenta tanto la presión de entrada del agua de alimentación ( $\mathrm{P}_{\mathrm{f}}{ }_{\mathrm{f}}$ como su temperatura promedio $(\bar{T})$ determinada en el paso anterior, se procederá a buscar las propiedades físicas mostradas en la Tabla 4 para este fluido. 
Tabla 4. Propiedades físicas requeridas para el agua de alimentación a la temperatura promedio determinada en el Paso 9.

\begin{tabular}{lcc}
\hline Propiedad & Símbolo & Unidades \\
\hline Densidad & $\rho_{\mathrm{f}}$ & $\mathrm{kg} / \mathrm{m}^{3}$ \\
Volumen específico & $\varepsilon_{\mathrm{f}}$ & $\mathrm{m}^{3} / \mathrm{kg}$ \\
Viscosidad cinemática & $\mathrm{V}_{\mathrm{f}}$ & $\mathrm{m}^{2} / \mathrm{s}$ \\
Viscosidad dinámica & $\mu_{\mathrm{f}}$ & $\mathrm{Pa} . \mathrm{s}$ \\
Conductividad térmica & $\mathrm{k}_{\mathrm{f}}$ & $\mathrm{W} / \mathrm{m} . \mathrm{K}$ \\
Número de Prandtl & $\mathrm{Pr}_{\mathrm{f}}$ & - \\
\hline Fuente: Elaboración propia & &
\end{tabular}

Paso 11. Área superficial de las aletas por $1 \mathrm{~m}$ de longitud del tubo para aletas helicoidales $\left(\mathrm{A}_{\mathrm{r} 1}\right)$ :

$$
A_{r 1}=\frac{\pi}{2} \cdot\left(D^{2}-d^{2}+2 \cdot D \cdot \delta_{r}\right) \cdot \frac{L_{r s}}{s_{r}} \cdot z
$$

Paso 12. Área superficial del tubo portador, no ocupada por las aletas, por $1 \mathrm{~m}$ de longitud del tubo aleteado $\left(\mathrm{A}_{\mathrm{t} 1}\right)$ :

$$
A_{t 1}=\pi \cdot d \cdot\left[L_{r s} \cdot\left(1-\frac{\delta_{r}}{s_{r}}\right) \cdot z+L_{t}\right]
$$

Paso 13. Área de la superficie externa por $1 \mathrm{~m}$ de longitud del tubo $\left(\mathrm{A}_{1}\right)$ :

$$
A_{1}=A_{r 1}+A_{t 1}
$$

Paso 14. Relación $A_{r} / A$ :

$$
\frac{A_{r}}{A}=\frac{A_{r 1}}{A_{1}}
$$

Paso 15. Relación $A_{t} / A$ :

$$
\frac{A_{t}}{A}=\frac{A_{t 1}}{A_{1}}
$$

Paso 16. Área superficial total del tubo portador aleteado por $1 \mathrm{~m}$ de longitud del tubo $\left(\mathrm{A}_{\mathrm{rct} 1}\right)$ :

$$
A_{r c t 1}=\pi \cdot d \cdot L_{r s}
$$

Paso 17. Área de la superficie interna por $1 \mathrm{~m}$ de longitud del tubo $\left(\mathrm{A}_{\mathrm{in} 1}\right)$ :

$$
A_{i n 1}=\pi \cdot d_{i n} \cdot L_{r s}
$$


Paso 18. Relación $A / A_{\text {in }}$ :

$$
\frac{A}{A_{i n}}=\frac{A_{1}}{A_{i n 1}}
$$

Paso 19. Coeficiente de la aleta del tubo $\left(\Psi_{\mathrm{r}}\right)$ :

$$
\psi_{r}=\frac{A}{A_{r c t}}=\frac{A_{1}}{A_{r c t 1}}
$$

Paso 20. Espaciado transversal del tubo $\left(S_{1}\right)$ :

$$
S_{1}=\frac{a}{z_{1}+0,5}
$$

Paso 21. Espaciado longitudinal del tubo $\left(\mathrm{S}_{2}\right)$ :

$$
S_{2}=\frac{\sqrt{3}}{2} \cdot S_{1}
$$

Paso 22. Definición del espaciado diagonal del tubo con un arreglo "equivalente" $\left(\mathrm{S}_{2}{ }_{2}\right)$.

Paso 23. Espaciado transversal relativo de los tubos en el haz $\left(\sigma_{1}\right)$ :

$$
\sigma_{1}=\frac{S_{1}}{d}
$$

Paso 24. Espaciado longitudinal relativo de los tubos $\left(\sigma_{2}\right)$ :

$$
\sigma_{2}=\frac{S_{2}}{d}
$$

Paso 25. Características del espaciado relativo:

$$
\begin{aligned}
& \sigma_{2}^{\prime}=\frac{S_{2}^{\prime}}{d} \\
& \frac{\sigma_{1}}{\sigma_{2}}=\frac{S_{1}}{S_{2}}
\end{aligned}
$$

Paso 26. Diámetro convencional del tubo aleteado $\left(\mathrm{d}_{\mathrm{cl}}\right)$ : 


$$
d_{c l}=d+\frac{2 \cdot l_{r} \cdot \delta_{r}}{s_{r}}
$$

Paso 27. Diámetro del haz $\left(\varphi_{\mathrm{cl}}\right)$ :

$$
\varphi_{c l}=\frac{S_{1}-d_{c l}}{S_{2}^{\prime}-d_{c l}}
$$

Paso 28. Área libre mínima (F):

$$
F=a \cdot b-z_{1} \cdot L_{c \cdot c r \cdot s} \cdot d_{c l}
$$

Paso 29. Velocidad del gas calculada $\left(\mathrm{u}_{\mathrm{g}}\right)$ :

$$
u_{g}=\frac{G_{g} \cdot \varepsilon_{g}}{F}
$$

Paso 30. Numero de comienzos del serpentín $\left(\mathrm{n}_{\mathrm{x}}\right)$.

Paso 31. Número de tubos conectados en paralelo $\left(\mathrm{Z}_{\mathrm{tcp}}\right)$ :

$$
z_{t c p}=n_{x} \cdot z_{1}
$$

Paso 32. Área de flujo para el paso del medio de transferencia de calor $(f)$ :

$$
f=z_{t c p} \cdot \frac{\pi \cdot d_{i n}^{2}}{4}
$$

Paso 33. Velocidad promedio del agua $\left(u_{f}\right)$ :

$$
u_{f}=\frac{G_{f} \cdot \varepsilon_{f}}{f}
$$

Paso 34. Eficiencia térmica ( $\Psi)$.

Paso 35. Resistencia térmica de la pared de los tubos bimetálicos $\left(\mathrm{R}_{\mathrm{T}}\right)$.

Paso 36. Parámetro de forma del haz (X):

Para un arreglo triangular de los tubos: 


$$
X=\frac{\sigma_{1}}{\sigma_{2}}-\frac{1,26}{\psi_{r}}-2
$$

Paso 37. Exponente n:

$$
n=0,7+0,08 \cdot \tanh (X)+0,005 \cdot \psi_{r}
$$

Paso 38. Factor $\mathrm{C}_{\mathrm{q}}$ :

$$
C_{q}=[1,36-\tanh (X)] \cdot\left(\frac{1,1}{\psi_{r}+8}-0,014\right)
$$

Paso 39. Factor $\mathrm{C}_{\mathrm{z}}$.

Paso 40. Coeficiente de transferencia de calor convectivo $\left(h_{c}\right)$ :

$$
h_{c}=1,13 \cdot C_{z} \cdot C_{q} \cdot \frac{k_{g}}{d} \cdot\left(\frac{u_{g} \cdot d}{v_{g}}\right)^{n} \cdot \operatorname{Pr}_{g}^{0,33}
$$

\section{Coeficiente de transferencia de calor reducido:}

Paso 41. Suposición del valor preliminar de la eficiencia teórica de la aleta (E').

Paso 42. Temperatura promedio de la aleta $\left(\mathrm{T}_{\mathrm{r}}\right)$ :

$$
T_{r}=\bar{\vartheta}-(\bar{\vartheta}-\bar{T}) \cdot E^{\prime}
$$

Paso 43. Conductividad térmica del material de la aleta $\left(k_{r}\right)$ a la temperatura promedio $\left(T_{r}\right)$ determinada en el paso anterior.

Paso 44. Parámetro de la aleta $(\beta)$ :

$$
\beta=\sqrt{\frac{2 \cdot h_{c}}{\delta_{r} \cdot k_{r}}}
$$

Paso 45. Altura convencional de la aleta $\left(l^{\prime}{ }_{r}\right)$ :

$$
l_{r}^{\prime}=l_{r} \cdot\left[1+\left(0,191+0,054 \cdot \frac{D}{d}\right) \cdot \ln \left(\frac{D}{d}\right)\right]
$$

Paso 46. Eficiencia teórica de la aleta (E): 


$$
E=\frac{\tanh \left(\beta \cdot l_{r}^{\prime}\right)}{\beta \cdot l_{r}^{\prime}}
$$

Paso 47. Comparación del valor de la eficiencia teórica de la aleta (E) determinado en el Paso 46 con el valor de eficiencia asumido preliminarmente en el Paso 41 (E').

En caso de existir una diferencia no superior a $\pm 0,02$ entre $E$ y E', se toma el valor de E determinado en el Paso 46. De lo contrario se vuelve a iterar.

Paso 48. Factor $\left(\Psi_{\mathrm{E}}\right)$ :

$$
\psi_{E}=1-0,016 \cdot\left(\frac{D}{d}-1\right) \cdot\left[1+\tanh \left(2 \cdot \beta \cdot l_{r}-1\right)\right]
$$

Paso 49. Coeficiente $\mu_{\mathrm{r}}$.

Paso 50. Coeficiente de transferencia de calor reducido desde el exterior de la superficie de calentamiento $\left(\mathrm{h}_{1 \mathrm{rdc}}\right)$ :

$$
h_{1 r d c}=\left(\frac{A_{r}}{A} \cdot E \cdot \mu_{r} \cdot \psi_{E}+\frac{A_{t}}{A}\right) \cdot h_{c}
$$

Coeficiente de transferencia de calor desde la pared hacia el medio interno

Paso 51. Número de Reynolds para el agua $\left(\operatorname{Re}_{\mathrm{f}}\right)$ :

$$
\operatorname{Re}_{f}=\frac{u_{f} \cdot d_{i n}}{v_{f}}
$$

Paso 52. Parámetro $\lambda$ :

$$
\lambda=1+\frac{900}{\operatorname{Re}_{f}}
$$

Paso 53. Parámetro $\zeta:$

$$
\zeta=\left(1,82 \cdot \log \left(\operatorname{Re}_{f}\right)-1,64\right)^{-2}
$$

Paso 54. Suposición del valor del área de la superficie exterior de transferencia de calor (A').

Paso 55. Suposición del coeficiente de transferencia de calor (h’2).

Paso 56. Área interior calculada ( $\left.\mathrm{A}^{\prime}{ }_{\mathrm{in}}\right)$ : 


$$
A_{i n}^{\prime}=\frac{A^{\prime}}{\left(\frac{A}{A_{i n}}\right)}
$$

Paso 57. Temperatura interior calculada $\left(T^{\prime}{ }_{i n}\right)$ :

$$
T^{\prime}{ }_{i n}=\bar{T}+\frac{Q \cdot 10^{3}}{A_{i n}^{\prime} \cdot h_{2}^{\prime}}
$$

Paso 58. Viscosidad dinámica del agua a $\mathrm{P}_{\mathrm{f}} \mathrm{y} T_{i n}^{\prime}\left(\mu_{\text {in }}\right)$.

Paso 59. Factor de corrección $\left(\mathrm{C}_{\mathrm{tem}}\right)$ :

Para el caso considerado donde el medio de transferencia de calor interno es líquido en descenso (agua) y que es calentado (el flujo de calor es dirigido desde la pared del tubo hacia el agua), el factor de corrección se determina mediante la siguiente ecuación:

$$
C_{\text {tem }}=\left(\frac{\mu_{f}}{\mu_{i n}}\right)^{0,11}
$$

Paso 60. Coeficiente de transferencia de calor desde la pared hacia el medio interno $\left(\mathrm{h}_{2}\right)$ :

$$
h_{2}=\frac{k_{f}}{d_{\text {in }}} \cdot\left[\frac{0,125 \cdot \zeta \cdot \operatorname{Re}_{f} \cdot \operatorname{Pr}_{f} \cdot C_{\text {tem }}}{\lambda+4,5 \cdot \zeta^{0.5} \cdot\left(\operatorname{Pr}_{f}^{0,666}-1\right)}\right]
$$

\section{Dimensiones del intercambiador:}

Paso 61. Coeficiente global de transferencia de calor (U):

$$
U=\frac{\psi}{\frac{A}{A_{i n}} \cdot \frac{1}{h_{2}}+\frac{A}{A_{i n}} \cdot R_{t}+\frac{1}{h_{1 r d c}}}
$$

Paso 62. Diferencia promedio de temperatura $(\Delta \mathrm{T})$ :

Para arreglo a contracorriente:

$$
\Delta T=\frac{\left(\vartheta^{\prime}-T^{\prime \prime}\right)-\left(\vartheta^{\prime \prime}-T^{\prime}\right)}{\ln \frac{\left(\vartheta^{\prime}-T^{\prime \prime}\right)}{\left(\vartheta^{\prime \prime}-T^{\prime}\right)}}
$$


Paso 63. Área de la superficie de transferencia de calor del intercambiador de calor (A):

$$
A=\frac{Q \cdot 10^{3}}{U \cdot \Delta T}
$$

Paso 64. Área de la superficie de transferencia de calor interna calculada $\left(\mathrm{A}_{\text {in }}\right)$ :

$$
A_{i n}=\frac{A}{\left(\frac{A}{A_{i n}}\right)}
$$

Paso 65. Temperatura interior calculada $\left(T_{\text {in }}\right)$ :

$$
T_{i n}=\bar{T}+\frac{Q \cdot 10^{3}}{A_{\text {in }} \cdot h_{2}}
$$

Paso 66. Comparación del valor de la temperatura interior $T_{\text {in }}$ calculado en el Paso 65 con el valor de la temperatura interior $\left(T^{\prime}{ }_{i n}\right)$ determinado en el Paso 57.

En caso de existir una diferencia no mayor a $\pm 1,00{ }^{\circ} \mathrm{C}$ entre $T_{i n}$ y $T^{\prime}{ }_{i n}$, se tomará el valor de $T_{\text {in }}$ determinado en el Paso 65. De lo contrario se vuelve a iterar.

Paso 67. Longitud total de los tubos aleteados ( $\sin \operatorname{codos})$ del intercambiador de calor $\left(\mathrm{L}_{\mathrm{rs}}\right)$ :

$$
L_{r s}=\frac{A}{A_{1}}
$$

Paso 68. Número total de tubos en el intercambiador de calor (z):

$$
z=\frac{L_{r s}}{L_{c . c r . s}}
$$

Paso 69. Numero de filas transversales de los tubos $\left(\mathrm{z}_{2}\right)$ :

$$
z_{2}=\frac{z}{z_{1}}
$$

Paso 70. Profundidad del conducto de gas (c):

$$
c=\left(z_{2}-1\right) \cdot S_{2}
$$


Paso 71. Número real de tubos en el intercambiador de calor $\left(z^{a}\right)$ :

$$
z^{a}=z_{1} \cdot z_{2}
$$

Paso 72. Longitud actual de los tubos aleteados del intercambiador de calor ( $\left.L_{r s}^{a}\right)$ :

$$
L_{r s}^{a}=L_{c \cdot c \cdot s} \cdot z^{a}
$$

\section{RESULTADOS Y DISCUSIÓN}

A continuación, se muestran los resultados obtenidos al aplicar la metodología de diseño del economizador.

\subsection{Coeficiente de transferencia de calor convectivo}

Paso 1. Definición de los datos iniciales de las corrientes:

La Tabla 5 expresa los valores de los datos iniciales de cada corriente involucrada en el sistema de transferencia de calor.

Tabla 5. Valores de los datos iniciales de cada corriente involucrada:

\begin{tabular}{lccc}
\hline Parámetro & Agua & Gases & Unidades \\
\hline Caudal másico & 1,200 & 1,857 & $\mathrm{~kg} / \mathrm{s}$ \\
Temperatura de entrada & 25 & 240 & ${ }^{\circ} \mathrm{C}$ \\
Temperatura de salida & - & 120 & ${ }^{\circ} \mathrm{C}$ \\
Presión de entrada & 0,5 & 0,1 & $\mathrm{MPa}$ \\
\hline Fuente: Elaboración propia & & &
\end{tabular}

Paso 2. Definición de los datos requeridos para el intercambiador de calor:

Como bien se estableció con anterioridad, el intercambiador tendrá tubos bimetálicos con arreglo triangular y aletas externas helicoidales, los cuales estarán conectados en forma de serpentín en dirección longitudinal con respecto al paso del aire. Los codos estarán ubicados por fuera del conducto de aire. En la Tabla 6 se muestran los valores de datos iniciales necesarios para el intercambiador de calor.

Tabla 6. Valores de los datos iniciales requeridos para el intercambiador de calor:

\begin{tabular}{lcccc}
\hline Parámetro & & Símbolo & Valor & Unidades \\
\hline & Tubos & & & \\
Diámetro interno & & $\mathrm{d}_{\text {in }}$ & 0,02786 & $\mathrm{~m}$ \\
Espesor de la pared & & $\delta^{\prime}$ & 0,00277 & $\mathrm{~m}$ \\
Diámetro externo & Aletas & $\mathrm{d}$ & 0,03340 & $\mathrm{~m}$ \\
& & $\mathrm{D}$ & 0,0604 & $\mathrm{~m}$ \\
Diámetro del tubo aleteado & & $\mathrm{l}_{\mathrm{r}}$ & 0,0135 & $\mathrm{~m}$ \\
Altura de la aleta & & $\mathrm{S}_{\mathrm{r}}$ & 0,0030 & $\mathrm{~m}$ \\
Espaciado de las aletas & & $\delta_{\mathrm{r}}$ & 0,0008 & $\mathrm{~m}$ \\
Espesor medio de las aletas & Otros datos & & & \\
& & $\mathrm{a}$ & 0,56 & $\mathrm{~m}$
\end{tabular}


Longitud de los segmentos aleteados de los tubos

Número de tubos en el haz

Longitud total de los segmentos calentados de los tubos no aleteados

Número de tubos en la fila transversal del haz

Numero de filas de tubos en el haz en dirección al flujo de gas

Longitud de los tubos dentro de los confines del conducto de gas

Resistencia térmica de contacto en la interface acero-aluminio

Fuente: Elaboración propia

Paso 3. Temperatura promedio de los gases de combustión $(\bar{\vartheta})$ :

$$
\bar{\vartheta}=\frac{\vartheta^{\prime}+\vartheta^{\prime \prime}}{2}=\frac{240+120}{2}=180^{\circ} \mathrm{C}
$$

Paso 4. Búsqueda de las propiedades físicas de los gases de combustión a la temperatura promedio determinada en el paso anterior:

Según (Pávlov et al., 1981; Green y Southard, 2019; Ruiz, 2012; Pis'mennyi et al., 2016), los gases de combustión tendrán los valores de las propiedades físicas que se expresan en la Tabla 7 a la temperatura media determinada en el paso anterior.

Tabla 7. Valores de las propiedades físicas de los gases de combustión:

\begin{tabular}{lccc}
\hline Propiedad & Símbolo & Valor & Unidades \\
\hline Densidad & $\rho_{\mathrm{g}}$ & 0,779 & $\mathrm{~kg} / \mathrm{m}^{3}$ \\
Volumen especifico & $\varepsilon_{\mathrm{g}}$ & 1,284 & $\mathrm{~m}^{3} / \mathrm{kg}$ \\
Viscosidad cinemática & $\mathrm{Vg}_{\mathrm{g}}$ & $2,99 \times 10^{-5}$ & $\mathrm{~m} / \mathrm{s}$ \\
Calor especifico & $\mathrm{Cp}_{\mathrm{g}}$ & 1,087 & $\mathrm{~kJ} / \mathrm{kg} . \mathrm{K}$ \\
Conductividad térmica & $\mathrm{kg}_{\mathrm{g}}$ & 0,0350 & $\mathrm{~W} / \mathrm{m} . \mathrm{K}$ \\
Número de Prandtl & $\mathrm{Pr}_{\mathrm{g}}$ & 0,679 & - \\
\hline Fuente: Elaboración propia & & &
\end{tabular}

Paso 5. Calor intercambiado (Q):

$$
Q=G_{g} \cdot C p_{g} \cdot\left(\vartheta^{\prime}-\vartheta^{\prime \prime}\right)=1,857 \cdot 1,087 \cdot(240-120)=242,227 k W
$$

Paso 6. Entalpía del agua a la entrada del intercambiador de calor (h'):

Tomando en cuenta una temperatura ( $\mathrm{T}$ ') y presión de alimentación del agua $\left(\mathrm{P}_{\mathrm{f}}{ }_{\mathrm{f}}\right)$ de $25{ }^{\circ} \mathrm{C}$ y $0,5 \mathrm{MPa}$, respectivamente, la entalpía del agua será de 105,298 kJ/kg, de acuerdo con (Wagner y Kretzschmar, 2019).

Paso 7. Entalpía del agua a la salida del intercambiador de calor (h’”):

Utilizando la ecuación (3) se obtiene:

$$
h^{\prime \prime}=h^{\prime}+\frac{Q}{G_{f}}=105,298+\frac{242,227}{1,200}=307,154 \mathrm{~kJ} / \mathrm{kg}
$$

Paso 8. Temperatura del agua a la salida del intercambiador de calor ( $\left.\mathrm{T}^{\prime \prime}\right)$ : 
Para una presión de alimentación ( $\mathrm{P}_{\mathrm{f}}$ ) de 0,5 MPa y una entalpía de salida (h') de 307,154 kJ/kg, el agua se calentará hasta una temperatura de $73,27^{\circ} \mathrm{C}$ según (Wagner y Kretzschmar, 2019).

Paso 9. Temperatura promedio del agua en el intercambiador de calor $(\bar{T})$ :

$$
\bar{T}=\frac{T^{\prime}+T^{\prime \prime}}{2}=\frac{25+73,27}{2}=49,135^{\circ} \mathrm{C}
$$

Paso 10. Propiedades físicas del agua de alimentación:

La Tabla 8 expresa los valores de las propiedades físicas del agua de alimentación determinados a la temperatura media $(\bar{T})$ calculada en el paso anterior, así como a la presión ( $\mathrm{P}_{\mathrm{f}}{ }_{\text {) de }}$ 0,5 MPa, de acuerdo con (ChemicaLogic, 2003; Wagner y Kretzschmar, 2019).

Tabla 8. Valores de las propiedades físicas del agua de alimentación:

\begin{tabular}{lccc}
\hline Propiedad & Símbolo & Valor & Unidades \\
\hline Densidad & $\rho_{\mathrm{f}}$ & 988,598 & $\mathrm{~kg} / \mathrm{m}^{3}$ \\
Volumen específico & $\varepsilon_{\mathrm{f}}$ & 0,00101 & $\mathrm{~m}^{3} / \mathrm{kg}$ \\
Viscosidad cinemática & $\mathrm{v}_{\mathrm{f}}$ & $5,614 \times 10^{-7}$ & $\mathrm{~m}^{2} / \mathrm{s}$ \\
Viscosidad dinámica & $\mu_{\mathrm{f}}$ & 0,000555 & $\mathrm{~Pa} . \mathrm{s}$ \\
Conductividad térmica & $\mathrm{k}_{\mathrm{f}}$ & 0,643 & $\mathrm{~W} / \mathrm{m} . \mathrm{K}$ \\
Número de Prandtl & $\mathrm{Pr}_{\mathrm{f}}$ & 3,609 & - \\
\hline Fuente: Elaboración propia & & &
\end{tabular}

Fuente: Elaboración propia

La Tabla 9 describe los resultados de los parámetros determinados en los Pasos 11 al 21.

Tabla 9. Resultados de los parámetros incluidos en los pasos 11-21:

\begin{tabular}{clccc}
\hline Paso & Parámetro & Símbolo & Valor & \multicolumn{2}{c}{ Unidades } \\
\hline 11 & $\begin{array}{l}\text { Área superficial de las aletas por 1 m de longitud del tubo } \\
\text { para aletas helicoidales }\end{array}$ & $\mathrm{A}_{\mathrm{r} 1}$ & 1,376 & $\mathrm{~m}^{2}$ \\
12 & $\begin{array}{l}\text { Área superficial del tubo portador, no ocupada por las aletas, } \\
\text { por 1 m de longitud del tubo aleteado }\end{array}$ & $\mathrm{At} 1$ & 0,077 & $\mathrm{~m}^{2}$ \\
& & & \\
13 & Área de la superficie externa por 1 m de longitud del tubo & $\mathrm{A}_{1}$ & 1,453 & $\mathrm{~m}^{2}$ \\
14 & Relación $A_{r} / A$ & $A_{r 1} / A_{1}$ & 0,947 & - \\
15 & Relación $A_{t} / A$ & $A_{t 1} / A_{1}$ & 0,053 & - \\
16 & Área superficial total del tubo portador aleteado por 1 m de & $\mathrm{A}_{\mathrm{rct} 1}$ & 0,105 & $\mathrm{~m}^{2}$ \\
& lónitud del tubo & $\mathrm{A}_{\text {in1 } 1}$ & 0,087 & $\mathrm{~m}^{2}$ \\
17 & Área de la superficie interna por 1 m de longitud del tubo & $A_{1} / A_{i n 1}$ & 16,701 & - \\
18 & Relación $A / A_{\text {in }}$ & $\Psi_{\mathrm{r}}$ & 13,838 & - \\
19 & Coeficiente de la aleta del tubo & $\mathrm{S}_{1}$ & 0,059 & $\mathrm{~m}$ \\
20 & Espaciado transversal del tubo & $\mathrm{S}_{2}$ & 0,051 & $\mathrm{~m}$ \\
\hline Fuente: Elaboración propia & & &
\end{tabular}

Paso 22. Definición del espaciado diagonal del tubo con un arreglo "equivalente" $\left(\mathrm{S}_{2}{ }_{2}\right)$.

Según (Pis'mennyi et al., 2016), el valor del espaciado del tubo diagonal con un arreglo "equivalente" $\left(\mathrm{S}_{2}{ }_{2}\right)$ es igual al espaciado del tubo transversal $\left(\mathrm{S}_{1}\right)$, por lo que: 
$\mathrm{S}_{2}{ }_{2}=\mathrm{S}_{1}=0,059 \mathrm{~m}$.

La Tabla 10 muestra los resultados de los parámetros calculados en los Pasos 23 al 29.

Tabla 10. Resultados de los parámetros incluidos en los pasos 23-29:

\begin{tabular}{rlccc}
\hline Paso & Parámetro & Símbolo & Valor & Unidades \\
\hline 23 & Espaciado transversal relativo de los tubos en el haz & $\sigma_{1}$ & 1,766 & - \\
24 & Espaciado longitudinal relativo de los tubos & $\sigma_{2}$ & 1,527 & - \\
25 & Características del espaciado relativo & $\sigma_{2}^{\prime}$ & 1,766 & - \\
& & $\sigma_{1} / \sigma_{2}$ & 1,156 & - \\
26 & Diámetro convencional del tubo aleteado & $\mathrm{d}_{\mathrm{cl}}$ & 0,041 & $\mathrm{~m}$ \\
27 & Diámetro del haz & $\varphi_{\mathrm{cl}}$ & 1 & $\mathrm{~m}$ \\
28 & Área libre mínima & $\mathrm{F}$ & 0,096 & $\mathrm{~m}^{2}$ \\
29 & Velocidad del gas calculada & $\mathrm{u}_{\mathrm{g}}$ & 24,837 & $\mathrm{~m} / \mathrm{s}$ \\
\hline Fuente: Elaboración propia & & &
\end{tabular}

Paso 30. Numero de comienzos del serpentín $\left(\mathrm{n}_{\mathrm{x}}\right)$ :

Para un arreglo triangular de tubos, $\mathrm{n}_{\mathrm{x}} \geq 2$ (Pis'mennyi et al., 2016), por lo que se tomará un valor inicial del $n_{x}=2$.

Paso 31. Número de tubos conectados en paralelo $\left(\mathrm{z}_{\mathrm{tcp}}\right)$ :

$$
z_{t c p}=n_{x} \cdot z_{1}=2 \cdot 9=18
$$

Paso 32. Área de flujo para el paso del medio de transferencia de calor $(f)$ :

$$
f=z_{t c p} \cdot \frac{\pi \cdot d_{i n}^{2}}{4}=18 \cdot \frac{3,14 \cdot(0,02786)^{2}}{4}=0,011 \mathrm{~m}^{2}
$$

Paso 33. Velocidad promedio del agua $\left(u_{f}\right)$ :

$$
u_{f}=\frac{G_{f} \cdot \varepsilon_{f}}{f}=\frac{1,2 \cdot 0,00101}{0,011}=0,110 \mathrm{~m} / \mathrm{s}
$$

Paso 34. Eficiencia térmica ( $\Psi)$ :

Tomando en cuenta los establecido por (Pis'mennyi et al., 2016), se toma un valor de $\Psi$ igual a 0,60 ya que el tipo de combustible quemado es petróleo y que el tipo de superficie de calentamiento es las etapas "calientes" de los economizadores.

Paso 35. Resistencia térmica de la pared de los tubos bimetálicos $\left(\mathrm{R}_{\mathrm{T}}\right)$ :

Para determinar la resistencia térmica de la pared de los tubos bimetálicos $\left(\mathrm{R}_{\mathrm{T}}\right)$, resulta posible despreciar los parámetros $\delta_{T}^{\prime} / k_{T}^{\prime}$ y $\delta^{\prime \prime}{ }_{T} / k^{\prime \prime}{ }_{T}$ debido a sus pequeños valores, por lo que puede asumirse que $\mathrm{R}_{\mathrm{T}} \approx$ $\mathrm{R}_{\mathrm{cont}}=0,000189 \mathrm{~m}^{2} . \mathrm{K} / \mathrm{W}$. 
Paso 36. Parámetro de forma del haz (X):

$$
X=\frac{\sigma_{1}}{\sigma_{2}}-\frac{1,26}{\psi_{r}}-2=1,156-\frac{1,26}{13,838}-2=-0,935
$$

Paso 37. Exponente n:

$$
\begin{aligned}
& n=0,7+0,08 \cdot \tanh (X)+0,005 \cdot \psi_{r} \\
& n=0,7+0,08 \cdot \tanh (-0,935)+0,005 \cdot 13,838 \\
& n=0,711
\end{aligned}
$$

Paso 38. Factor $\mathrm{C}_{\mathrm{q}}$ :

$$
\begin{aligned}
& C_{q}=[1,36-\tanh (X)] \cdot\left(\frac{1,1}{\psi_{r}+8}-0,014\right) \\
& C_{q}=[1,36-\tanh (-0,935)] \cdot\left(\frac{1,1}{13,838+8}-0,014\right) \\
& C_{q}=0,075
\end{aligned}
$$

Paso 39. Factor $\mathrm{C}_{\mathrm{z}}$ :

Tomando en cuenta que $\sigma_{1} / \sigma_{2}<2$ y z $2>8$, se toma $C_{z}=1,0$ (Pis'mennyi et al., 2016).

Paso 40. Coeficiente de transferencia de calor convectivo $\left(h_{c}\right)$ :

$$
\begin{aligned}
& h_{c}=1,13 \cdot C_{z} \cdot C_{q} \cdot \frac{k_{g}}{d} \cdot\left(\frac{u_{g} \cdot d}{v_{g}}\right)^{n} \cdot \operatorname{Pr}_{g}^{0,33} \\
& h_{c}=1,13 \cdot 1,0 \cdot 0,075 \cdot \frac{0,0350}{0,03340} \cdot\left(\frac{24,837 \cdot 0,03340}{0,0000299}\right)^{0,711} \cdot(0,679)^{0,33} \\
& h_{c}=112,633 \mathrm{~W} / \mathrm{m}^{2} \cdot K
\end{aligned}
$$

\subsection{Coeficiente de transferencia de calor reducido}

Paso 41. Suposición del valor preliminar de la eficiencia teórica de la aleta (E’).

Se asume un valor para E' de 0,92, según recomendaciones de (Pis'mennyi et al., 2016).

Paso 42. Temperatura promedio de la aleta $\left(\mathrm{T}_{\mathrm{r}}\right)$ : 


$$
\begin{aligned}
T_{r} & =\bar{\vartheta}-(\bar{\vartheta}-\bar{T}) \cdot E^{\prime}=180-(180-49,135) \cdot 0,92 \\
T_{r} & =59,604^{\circ} \mathrm{C}
\end{aligned}
$$

Paso 43. Conductividad térmica del material de la aleta $\left(\mathrm{k}_{\mathrm{r}}\right)$ a la temperatura promedio $\left(\mathrm{T}_{\mathrm{r}}\right)$ determinada en el paso anterior:

Según (Green y Southard, 2019), la conductividad térmica del aluminio a $\mathrm{T}_{\mathrm{r}}=59,604{ }^{\circ} \mathrm{C}$ es de 262,194 $\mathrm{W} / \mathrm{m} . \mathrm{K}$.

Paso 44. Parámetro de la aleta $(\beta)$ :

$$
\beta=\sqrt{\frac{2 \cdot h_{c}}{\delta_{r} \cdot k_{r}}}=\sqrt{\frac{2 \cdot 112,633}{0,0008 \cdot 262,194}}=32,775
$$

Paso 45. Altura convencional de la aleta $\left(l_{r}^{\prime}\right)$ :

$$
\begin{aligned}
& l_{r}^{\prime}=l_{r} \cdot\left[1+\left(0,191+0,054 \cdot \frac{D}{d}\right) \cdot \ln \left(\frac{D}{d}\right)\right] \\
& l_{r}^{\prime}=0,0135 \cdot\left[1+\left(0,191+0,054 \cdot \frac{0,0604}{0,03340}\right) \cdot \ln \left(\frac{0,0604}{0,03340}\right)\right] \\
& l_{r}^{\prime}=0,016 \mathrm{~m}
\end{aligned}
$$

Paso 46. Eficiencia teórica de la aleta (E):

$$
\begin{aligned}
& E=\frac{\tanh \left(\beta \cdot l^{\prime}{ }_{r}\right)}{\beta \cdot l^{\prime}{ }_{r}}=\frac{\tanh (32,775 \cdot 0,016)}{32,775 \cdot 0,016} \\
& E=0,917
\end{aligned}
$$

Paso 47. Comparación del valor de la eficiencia teórica de la aleta (E) determinado en el Paso 46 con el valor de eficiencia asumido preliminarmente en el Paso 41 (E').

Considerando el valor de E calculado (0,917), este se encuentra dentro del rango de $\pm 0,02$ establecido con respecto al valor de valor preliminar de la eficiencia teórica de la aleta (E') asumido en el Paso 41, por lo que se empleará éste valor calculado de E para los cálculos posteriores, no resultando necesario efectuar iteración.

Paso 48. Factor $\Psi_{\mathrm{E}}$ : 


$$
\begin{aligned}
& \psi_{E}=1-0,016 \cdot\left(\frac{D}{d}-1\right) \cdot\left[1+\tanh \left(2 \cdot \beta \cdot l_{r}-1\right)\right] \\
& \psi_{E}=1-0,016 \cdot\left(\frac{0,0604}{0,0334}-1\right) \cdot[1+\tanh (2 \cdot 32,775 \cdot 0,0135-1)] \\
& \psi_{E}=0,989
\end{aligned}
$$

Paso 49. Coeficiente $\mu_{\mathrm{r}}$ :

El valor del coeficiente $\mu_{\mathrm{r}}$ toma el valor de 1,0 para aletas con un espesor aproximadamente constante (Pis'mennyi et al., 2016).

Paso 50. Coeficiente de transferencia de calor reducido desde el exterior de la superficie de calentamiento $\left(\mathrm{h}_{1 \mathrm{rdc}}\right)$ :

$$
\begin{aligned}
& h_{1 r d c}=\left(\frac{A_{r}}{A} \cdot E \cdot \mu_{r} \cdot \psi_{E}+\frac{A_{t}}{A}\right) \cdot h_{c} \\
& h_{1 r d c}=(0,947 \cdot 0,917 \cdot 1,0 \cdot 0,989+0,053) \cdot 112,633 \\
& h_{1 r d c}=103,830 \mathrm{~W} / \mathrm{m}^{2} . K
\end{aligned}
$$

3.3. Coeficiente de transferencia de calor desde la pared hacia el medio interno

Paso 51. Número de Reynolds para el agua $\left(\mathrm{Re}_{\mathrm{f}}\right)$ :

$$
\operatorname{Re}_{f}=\frac{u_{f} \cdot d_{i n}}{v_{f}}=\frac{0,110 \cdot 0,02786}{5,614 \cdot 10^{-7}}=5458,852
$$

Paso 52. Parámetro $\lambda$ :

$$
\lambda=1+\frac{900}{\operatorname{Re}_{f}}=1+\frac{900}{5458,852}=1,165
$$

Paso 53. Parámetro $\zeta:$

$$
\begin{aligned}
& \zeta=\left(1,82 \cdot \log \left(\operatorname{Re}_{f}\right)-1,64\right)^{-2}=(1,82 \cdot \log (5458,852)-1,64)^{-2} \\
& \zeta=0,037
\end{aligned}
$$

Paso 54. Suposición del valor del área de la superficie exterior de transferencia de calor (A'):

Se asume un valor de $A^{\prime}=50 \mathrm{~m}^{2}$.

Paso 55. Suposición del coeficiente de transferencia de calor $\left(\mathrm{h}_{2}{ }_{2}\right)$ : 
Se asume un valor de h' ${ }_{2}$ de $1800 \mathrm{~W} / \mathrm{m}^{2} . \mathrm{K}$.

Paso 56. Área interior calculada ( $\left.\mathrm{A}^{\prime}{ }_{\text {in }}\right)$ :

$$
A_{\text {in }}^{\prime}=\frac{A^{\prime}}{\left(\frac{A}{A_{\text {in }}}\right)}=\frac{50}{16,701}=2,994 \mathrm{~m}^{2}
$$

Paso 57. Temperatura interior calculada $\left(T^{\prime}{ }_{i n}\right)$ :

$$
T^{\prime}{ }_{\text {in }}=\bar{T}+\frac{Q \cdot 10^{3}}{A_{\text {in }}^{\prime} \cdot h_{2}^{\prime}}=49,135+\frac{242,227 \cdot 10^{3}}{2,994 \cdot 1800}=94,082^{\circ} \mathrm{C}
$$

Paso 58. Viscosidad dinámica del agua a $\mathrm{P}_{\mathrm{f}}^{\prime} \mathrm{y} T^{\prime}{ }_{i n}\left(\mu_{\mathrm{in}}\right)$.

Para un valor de $\mathrm{P}_{\mathrm{f}}$ de $0,5 \mathrm{MPa}$ y de $T^{\prime}{ }_{\text {in }}$ igual a $94,082^{\circ} \mathrm{C}$, se tiene que la viscosidad del agua $\left(\mu_{\text {in }}\right)$ es de 0,000300 Pa.s (ChemicaLogic, 2003).

Paso 59. Factor de corrección $\left(\mathrm{C}_{\mathrm{tem}}\right)$ :

$$
C_{\text {tem }}=\left(\frac{\mu_{f}}{\mu_{\text {in }}}\right)^{0,11}=\left(\frac{0,000555}{0,000300}\right)^{0,11}=1,070
$$

Paso 60. Coeficiente de transferencia de calor desde la pared hacia el medio interno $\left(\mathrm{h}_{2}\right)$ :

$$
\begin{aligned}
& h_{2}=\frac{k_{f}}{d_{\text {in }}} \cdot\left[\frac{0,125 \cdot \zeta \cdot \operatorname{Re}_{f} \cdot \operatorname{Pr}_{f} \cdot C_{t e m}}{\lambda+4,5 \cdot \zeta^{0,5} \cdot\left(\operatorname{Pr}_{f}^{0,666}-1\right)}\right] \\
& h_{2}=\frac{0,643}{0,02786} \cdot\left[\frac{0,125 \cdot 0,037 \cdot 5458,852 \cdot 3,609 \cdot 1,070}{1,165+4,5 \cdot 0,037^{0,5} \cdot\left(3,609^{0,666}-1\right)}\right] \\
& h_{2}=964,073 \mathrm{~W} / \mathrm{m}^{2} \cdot K
\end{aligned}
$$

\subsection{Dimensiones del intercambiador}

Paso 61. Coeficiente global de transferencia de calor (U): 


$$
\begin{aligned}
& U=\frac{\psi}{\frac{A}{A_{i n}} \cdot \frac{1}{h_{2}}+\frac{A}{A_{i n}} \cdot R_{t}+\frac{1}{h_{1 r d c}}} \\
& U=\frac{0,60}{16,701 \cdot \frac{1}{964,073}+16,701 \cdot 0,000189+\frac{1}{103,830}} \\
& U=19,947 \mathrm{~W} / \mathrm{m}^{2} \cdot K
\end{aligned}
$$

Paso 62. Diferencia promedio de temperatura $(\Delta \mathrm{T})$ :

$$
\Delta T=\frac{\left(\vartheta^{\prime}-T^{\prime \prime}\right)-\left(\vartheta^{\prime \prime}-T^{\prime}\right)}{\ln \frac{\left(\vartheta^{\prime}-T^{\prime \prime}\right)}{\left(\vartheta^{\prime \prime}-T^{\prime}\right)}}=\frac{(240-73,27)-(120-25)}{\ln \frac{(240-73,27)}{(120-25)}}=127,63^{\circ} \mathrm{C}
$$

Paso 63. Área de la superficie de transferencia de calor del intercambiador de calor (A):

$$
A=\frac{Q \cdot 10^{3}}{U \cdot \Delta T}=\frac{242,227 \cdot 10^{3}}{19,947 \cdot 127,63}=95,146 \mathrm{~m}^{2}
$$

Paso 64. Área de la superficie de transferencia de calor interna calculada $\left(\mathrm{A}_{\text {in }}\right)$ :

$$
A_{\text {in }}=\frac{A}{\left(\frac{A}{A_{\text {in }}}\right)}=\frac{95,146}{16,701}=5,697 \mathrm{~m}^{2}
$$

Paso 65. Temperatura interior calculada $\left(T_{i n}\right)$ :

$$
T_{\text {in }}=\bar{T}+\frac{Q \cdot 10^{3}}{A_{\text {in }} \cdot h_{2}}=49,135+\frac{242,227 \cdot 10^{3}}{5,697 \cdot 964,073}=93,238
$$

Paso 66. Comparación del valor de la temperatura interior $T_{i n}$ calculado en el Paso 65 con el valor de la temperatura interior $\left(T^{\prime}{ }_{i n}\right)$ determinado en el Paso 57.

Tomando en cuenta el valor de $T_{\text {in }}$ determinado en el paso anterior $\left(93,238^{\circ} \mathrm{C}\right)$, se puede considerar aceptable ya que se encuentra dentro del rango de $\pm 1,00^{\circ} \mathrm{C}$ establecido previamente con respecto al valor de $T^{\prime}{ }_{\text {in }}$ determinado en el Paso $57\left(94,082^{\circ} \mathrm{C}\right)$. De esta manera no resulta necesario iterar.

La Tabla 11 presenta los resultados de los parámetros incluidos en los Pasos 67 al 72. 
Tabla 11. Resultados de los parámetros incluidos en los Pasos 67-72.

\begin{tabular}{clccc}
\hline Paso & Parámetro & Símbolo & Valor & Unidades \\
\hline 67 & Longitud total de los tubos aleteados (sin codos) del & $\mathrm{L}_{\mathrm{rs}}$ & 65,482 & $\mathrm{~m}$ \\
& $\begin{array}{l}\text { intercambiador de calor } \\
68\end{array}$ & $\mathrm{z}$ & 131 & \\
69 & Numero total de tubos en el intercambiador de calor & $\mathrm{z}_{2}$ & $16^{\ddagger}$ & \\
70 & Profundidad del conducto de gas & $\mathrm{c}$ & 0,765 & $\mathrm{~m}$ \\
71 & Número real de tubos en el intercambiador de calor & $z^{a}$ & 144 & \\
72 & $\begin{array}{l}\text { Longitud actual de los tubos aleteados del } \\
\text { intercambiador de calor }\end{array}$ & $L_{r s}^{a}$ & 72 & $\mathrm{~m}$ \\
\hline
\end{tabular}

ॠ Se redondeó hasta el valor par más próximo, según recomendación de (Pis'mennyi et al., 2016)

Fuente: Elaboración propia

Tomando en cuenta los resultados obtenidos, el agua se calentará hasta una temperatura de $73,27{ }^{\circ} \mathrm{C}$, la velocidad lineal del gas y del agua alcanzaron valores de $24,837 \mathrm{~m} / \mathrm{s}$ y $0,110 \mathrm{~m} / \mathrm{s}$, respectivamente, y la tasa de intercambio de calor (Q) alcanzó un valor de $242,227 \mathrm{~kW}$. El agua fluirá bajo régimen turbulento considerando el valor del número de Reynolds obtenido para este fluido $(5458,852)$, mientras que la eficiencia teórica calculada de la aleta (E) tuvo un valor de 0,917, la cual se encuentra dentro del rango de $\pm 0,02$ establecido con relación al valor preliminar asumido en el Paso 41 para la eficiencia teórica de la aleta (E'), no siendo necesario efectuar la iteración posterior.

Por su parte, la temperatura interior $\left(T_{i n}\right.$ ) calculada en el Paso 65 tuvo un valor de $93,238{ }^{\circ} \mathrm{C}$, el cual se encuentra dentro del intervalo de $\pm 1,00{ }^{\circ} \mathrm{C}$ establecido en comparación con el valor de la temperatura interior $\left(T^{\prime}{ }_{i n}\right)$ calculado en el Paso $57\left(94,082^{\circ} \mathrm{C}\right)$, por lo que no resulta necesario iterar.

El coeficiente de transferencia de calor convectivo $\left(h_{c}\right)$ tuvo un valor de $112,633 \mathrm{~W} / \mathrm{m}^{2} . \mathrm{K}$, mientras que tanto el coeficiente de transferencia de calor reducido $\left(\mathrm{h}_{1 \mathrm{rdc}}\right.$ ) como el coeficiente de transferencia de calor desde la pared hacia el medio interno $\left(\mathrm{h}_{2}\right)$ presentaron valores calculados de 103,830 y $964,073 \mathrm{~W} / \mathrm{m}^{2} . \mathrm{K}$, respectivamente.

Se obtuvo como resultado un valor del coeficiente global de transferencia de calor (U) de 19,947 W/m².K, una diferencia promedio de temperatura $(\Delta \mathrm{T})$ de $127,63^{\circ} \mathrm{C}$ y un área de la superficie de transferencia de calor del intercambiador de calor (A) de $95,146 \mathrm{~m}^{2}$.

El economizador tendrá una longitud de los tubos aleteados $\left(\mathrm{L}_{\mathrm{rs}}\right)$ de 65,482 $\mathrm{m}$, un número total de tubos (z) de 131, un número de filas transversales de los tubos $\left(\mathrm{z}_{2}\right)$ de 16, una profundidad del conducto del gas (c) de $0,765 \mathrm{~m}$, un número real de tubos $\left(z^{a}\right)$ de 144 y una longitud actual de los tubos aleteados $\left(L_{r s}^{a}\right)$ igual a $72 \mathrm{~m}$.

En un estudio semejante, el autor (López, 2017) diseño un economizador siguiendo la metodología presentada por (Pysmenny et al., 2007), la cual es muy similar a la empleada en este artículo. En este trabajo la temperatura inicial y el caudal másico de los gases de combustión es de $274{ }^{\circ} \mathrm{C} \mathrm{y} 0,654 \mathrm{~kg} / \mathrm{s}$, respectivamente, mientras que para el agua de alimentación la temperatura y presión inicial es de $64{ }^{\circ} \mathrm{C} \mathrm{y}$ $0,552 \mathrm{MPa}$ respectivamente, con un caudal másico de $0,387 \mathrm{~kg} / \mathrm{s}$, lográndose que la temperatura del agua se incremente hasta los $127,4{ }^{\circ} \mathrm{C}$. Se obtuvieron además otros resultados tales como el coeficiente convectivo $\left(23,91 \mathrm{~W} / \mathrm{m}^{2} . \mathrm{K}\right)$, el coeficiente de convección relativo $\left(20,587 \mathrm{~W} / \mathrm{m}^{2} . \mathrm{K}\right)$, el coeficiente de convección de la pared hacia el fluido interno $\left(2488,65 \mathrm{~W} / \mathrm{m}^{2} . \mathrm{K}\right)$, el coeficiente global de transferencia de calor $\left(11,876 \mathrm{~W} / \mathrm{m}^{2} . \mathrm{K}\right)$, el área de la superficie de intercambio de calor del economizador $\left(105,13 \mathrm{~m}^{2}\right)$, la diferencia promedio de temperatura $\left(82,75^{\circ} \mathrm{C}\right)$, la cantidad de tubos longitudinales (14), la longitud total de los tubos aleteados del economizador $(50 \mathrm{~m})$ y la profundidad del conducto de gases $(1,162 \mathrm{~m})$. 


\section{CONCLUSIONES}

1. El agua se calentará hasta una temperatura de $73,27^{\circ} \mathrm{C}$, mientras que la tasa de intercambio de calor alcanzó un valor de 242,227 kW.

2. La eficiencia teórica calculada de la aleta tuvo un valor de 0,917 .

3. El coeficiente de transferencia de calor convectivo tuvo un valor de $112,633 \mathrm{~W} / \mathrm{m}^{2} . \mathrm{K}$, el coeficiente de transferencia de calor reducido presentó un valor de $103,830 \mathrm{~W} / \mathrm{m}^{2} . \mathrm{K}$, y el coeficiente de transferencia de calor desde la pared hacia el medio interno exhibió un valor de $964,073 \mathrm{~W} / \mathrm{m}^{2} . \mathrm{K}$.

4. Se obtuvo un valor del coeficiente global de transferencia de calor de $19,947 \mathrm{~W} / \mathrm{m}^{2} . \mathrm{K}$, una diferencia promedio de temperatura de $127,63{ }^{\circ} \mathrm{C}$ y un área de la superficie de transferencia de calor del intercambiador de calor de $95,146 \mathrm{~m}^{2}$.

5. El economizador tendrá una longitud de los tubos aleteados de $65,482 \mathrm{~m}$, un número total de tubos de 131, un número de filas transversales de los tubos de 16, una profundidad del conducto del gas de $0,765 \mathrm{~m}$, un número real de tubos de 144 y una longitud actual de los tubos aleteados igual a $72 \mathrm{~m}$.

\section{REFERENCIAS}

Armijo, J., y Salas, G. (2008). Ahorro de energía en una caldera utilizando economizadores. Rev. Per. Quím. Ing. Quím., 11 (1), 42-46.

Brumerčík, F., Sojcak, D., Lukáč, M., Nieoczym, A., and Wierzbicki, S. (2016). Modeling of flow and temperature field in an economizer. Applied Computer Science, 12 (2), 63-73.

ChemicaLogic. (2003). Thermodynamic and Transport Properties of Water and Steam (Version 2.0). Burlington, USA: ChemicaLogic Corporation.

Chopade, M. M. (2018). Therma Design of Economizer for Waste Heat Recovery Boiler. International Journal of Engineering Research \& Technology, 7 (10), 96-102.

Chucuya, R. C. (2017). Ahorro de energía primaria mediante precalentamiento del aire y agua en una unidad de generación de vapor. INGnosis, 3 (2), 365-375.

Farthing, D. C. (2008). Improving Boiler Room Efficiencies - Economizers. Oklahoma, USA: Federal Corporation.

Green, D. W., and Southard, M. Z. (2019). Perry's Chemical Engineers' Handbook (9th ed.). New York, USA: McGraw-Hill.

Guiot, J. P., y Ramos, D. L. (2018). Diseño de un economizador para una caldera de 500 BHP. (Tesis de Diploma), Fundación Universidad de América, Bogotá, D.C., Colombia.

Jatola, R., Yadav, G., Jain, M. L., and More, B. (2017). Performance Analysis of Economizer Using Different Material of Tubes. International Research Journal of Engineering and Technology, 4 (4), 23892391.

Khoirul, A., and Firman, M. (2019). Design and Optimization of Boiler Economizer for Laundry Industry Using Taguchi Method. Advances in Social Science, Education and Humanities Research, 436, 533-537. 
López, H. A. (2017). Captar energía de gases de escape mediante un economizador para mejorar el rendimiento de una caldera pirotubular de 200 BHP. (Tesis de Diploma), Universidad Nacional del Centro del Perú, Huancayo, Perú.

Martić, I., Budimir, S., Mitrović, N., Maslarević, A., and Marković, M. (2015). Application and design of an economizer for waste heat recovery in a cogeneration plant. Thermal Science, 20 (4), 1355-1362. doi:10.2298/TSCI141113211M.

Melendez, E. R. (2005). Cálculo, diseño e instalación de un economizador para una caldera acuotubular de 2900 BHP. (Tesis de Diploma), Universidad Nacional del Santa, Nuevo Chimbote, Perú.

Morales, M. E., y Veintimilla, E. (2007). Diseño de un recuperador de calor para aprovechar la disponibilidad de los gases de combustión y calentar el colchón de agua del tanque de lavado de petróleo. (Tesis de Diploma), Escuela Politécnica Nacional, Quito, Ecuador.

Oelker, A. (1999). Economizadores. Revista InduAmbiente, 7 (37), 1-6.

Oelker, A. (2000). Análisis de la eficiencia en calderas. Santiago de Chile, Chile: Thermal Engineering, LTDA.

Pacheco, R. A., y Vargas, D. G. (2021). Recuperación de gases de caldera 500 BHP para calentamiento de agua en una máquina removedora de piel de pescado-Empresa Conservera la Chimbotana S.A.C. (Tesis de Diploma), Universidad Nacional del Santa, Nuevo Chimbote, Perú.

Pávlov, K. F., Romankov, P. G., y Noskov, A. A. (1981). Problemas y ejemplos para el curso de operaciones básicas y aparatos en tecnología química. Moscú, Rusia: Editorial Mir.

Pis'mennyi, E., Polupan, G., Carvajal, I., Sanchez, F., and Pioro, I. (2016). Handbook for transversely finned tube heat exchanger design. London, UK: Academic Press.

Polupan, G., Jarquin, G., Carvajal, I., y Pysmenny, Y. (2011). Desarrollo de Recuperadores de Energía para Calderas Basados en Elementos Bifásicos. Información Tecnológica, 22 (5), 83-90. doi:10.4067/S0718-07642011000500011.

Pysmenny, Y., Polupan, G., Carvajal, I., y Sánchez, F. (2007). Manual para el cálculo de intercambiadores de calor y bancos de tubos aletados. Barcelona, España: Editorial Reverté.

Restrepo, Á. H., Burbano, J. C., y Carranza, Y. A. (2006). Estudio de la factibilidad técnico-económica para la recuperación de calores residuales en la Empresa Manufacturas Carolina S.A. Scientia et Technica, XII (31), 79-84.

Ruiz, C. M. (2012). Análisis energético de caldera bagacera marca Babcock \& Wilcox: Propuesta de instalacion de economizador. (Tesis de Diploma), Universidad Veracruzana, Veracruz, México.

Trojan, M., and Granda, M. (2018). Modeling of the boiler economizer. MATEC Web of Conferences, 240, 1-7. doi:10.1051/matecconf/201824005034.

Wagner, W., and\& Kretzschmar, H.-J. (2019). International Steam Tables (3rd ed.). Berlin, Germany: Springer-Verlag GmbH Germany. 
Zarifi, S., and Moghaddam, M. M. (2020). Utilizing finned tube economizer for extending the thermal power rate of TEG CHP system. Energy, 202, 1-13.

\section{SEMBLANZA DE LOS AUTORES}

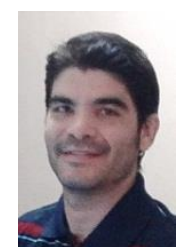

Amaury Pérez Sánchez: Obtuvo el grado de Ingeniero Químico en la Universidad de Camagüey, Cuba en el año 2009, donde actualmente es profesor instructor e investigador auxiliar. En estos momentos se encuentra estudiando un Máster en Biotecnología en el Centro de Ingeniería Genética y Biotecnología de Camagüey. Trabaja en líneas de investigación vinculadas fundamentalmente con el diseño de equipos de transferencia de calor y masa, simulación y optimización de procesos y operaciones de la industria química mediante el empleo de simuladores tales como SuperPro Designer ${ }^{\circledR}$ y ChemCAD ${ }^{\circledR}$, y la evaluación técnico-económica de procesos y plantas biotecnológicas.

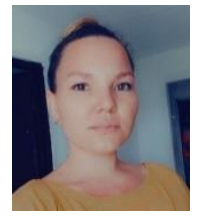

Noemí Acosta Pérez: Obtuvo el grado de Ingeniero Químico en la Universidad de Camagüey, Cuba en el año 2020. Se desempeña profesionalmente como Especialista Adiestrada del Laboratorio FísicoQuímico de la Empresa Azucarera Camagüey (AZCUBA), Cuba. Trabaja en líneas de investigación relacionadas con el diseño de equipos de transferencia de calor y masa, determinación cuantitativa de muestras de procesos de la industria azucarera, y evaluación termodinámica de operaciones y etapas de la rama energética.

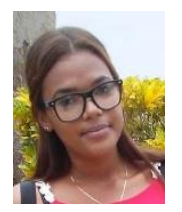

Greisy Ivety Valero Almanza: Obtuvo el grado de Ingeniero Quimico en la Universidad de Camagüey, Cuba en el año 2019. Se desempeña profesonalmente como Especialista de Ciencia, Tecnología y Medio Ambiente en el Departamento de Gestión Ambiental del Centro de Ingenieria Ambiental de Camagüey. Su área de investigación comprende la realización de estudios de riesgos mediombientales en empresas y fábricas quimicas, gestión medioambiental de residuales y desechos químicos, análisis fisico-quimico de aguas y aguas residuales, asi como tambien el diseño y evaluación de equipos de transferencia de calor y masa.

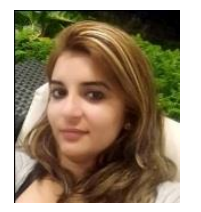

Elizabeth Ranero González. Obtuvo el grado de Ingeniero Químico en la Universidad de Camagüey, Cuba en el año 2016. Se desempeña profesionalmente como Profesor Instructor en la Universidad de Camagüey, Cuba. Su área de investigación abarca el diseño y evaluación de equipos y procesos de transferencia de calor y masa, evaluación termodinámica de procesos químicos, y la simulación de procesos y sistemas biotecnológicos.

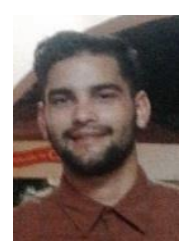

Eddy Javier Pérez Sánchez: Obtuvo el grado de Ingeniero Químico en la Universidad de Camagüey, Cuba en el año 2016. Se desempeña profesionalmente en la Empresa de Servicios Automotores S.A., en el Departamento de Dirección Comercial. Trabaja en líneas de investigación relacionadas con el diseño, evaluación y/o operación de equipos de transferencia de calor y masa, asi como también la simulación de procesos petroquímicos y biotecnológicos. 